\title{
ON CENTRALIZERS OF ELEMENTS OF GROUPS ACTING ON TREES WITH INVERSIONS
}

\author{
R. M. S. MAHMOOD
}

Received 2 May 2002

\begin{abstract}
A subgroup $H$ of a group $G$ is called malnormal in $G$ if it satisfies the condition that if $g \in G$ and $h \in H, h \neq 1$ such that $g h g^{-1} \in H$, then $g \in H$. In this paper, we show that if $G$ is a group acting on a tree $X$ with inversions such that each edge stabilizer is malnormal in $G$, then the centralizer $C(g)$ of each nontrivial element $g$ of $G$ is in a vertex stabilizer if $g$ is in that vertex stabilizer. If $g$ is not in any vertex stabilizer, then $C(g)$ is an infinite cyclic if $g$ does not transfer an edge of $X$ to its inverse. Otherwise, $C(g)$ is a finite cyclic of order 2.
\end{abstract}

2000 Mathematics Subject Classification: 20F65, 20E07, 20E08.

1. Introduction. There are many groups with the property that centralizers of nontrivial elements are cyclic. For example, the centralizers of the nontrivial elements (see [3, Problem 7, page 42]) of free groups are infinite cyclic. It has been shown in [3, Problem 28, page 196] that the centralizers of the nontrivial elements of the free product of groups are in a conjugate of a factor or infinite cyclic. In [1, Theorem 1], Karrass and Solitar proved that the centralizers of nontrivial elements of the free product of two groups with a malnormal amalgamated subgroup are in a conjugate of a factor or infinite cyclic. In this paper, we generalize such results to groups acting on trees with inversions as follows. If $G$ is a group acting on a tree $X$ with inversions such that the stabilizer $G_{X}$ of every edge $x$ of $X$ is a malnormal subgroup in $G$, then the centralizers $C(g)$ of every nontrivial element $g$ of $G$ is in a vertex stabilizer $G_{v}$ of a vertex $v$ of $X$ if $g$ is in $G_{v}$. If $g$ is not in any vertex stabilizer of $G$, then $C(g)$ is an infinite cyclic subgroup of $G$ if $g$ does not transfer any edge of $X$ to its inverse. Otherwise, $C(g)$ is a finite cyclic subgroup of $G$ of order 2 .

This paper is divided into five sections. In Section 2, we introduce the concepts of graphs and the actions of groups on graphs. In Section 3, we have a summary of the structure of groups acting on trees with inversions and elementary results. In Section 4, we discuss the structure of the centralizers of the elements of groups acting on trees with inversions. Section 5 is an application of the results in Section 4.

2. Basic concepts. We begin by giving preliminary definitions. 
By a graph $X$, we understand a pair of disjoint sets $V(X)$ and $E(X)$ with $V(X)$ nonempty together with a mapping $E(X) \rightarrow V(X) \times V(X), y \rightarrow(o(y), t(y))$, and a mapping $E(X) \rightarrow E(X), y \rightarrow \bar{y}$, satisfying the conditions that $\overline{\bar{y}}=y$ and $o(\bar{y})=t(y)$, for all $y \in E(X)$. The case $\bar{y}=y$ is possible for some $y \in E(X)$. For $y \in E(X), o(y)$ and $t(y)$ are called the ends of $y$, and $\bar{y}$ is called the inverse of $y$. There are obvious definitions of subgraphs, trees, morphisms of graphs, and $\operatorname{Aut}(X)$, the set of all automorphisms of the graph $X$ which is a group under the composition of morphisms of graphs. For more details, see $[4,5]$. We say that a group $G$ acts on a graph $X$ if there is a group homomorphism $\phi: G \rightarrow \operatorname{Aut}(X)$. If $x \in X$ (vertex or edge) and $g \in G$, we write $g(x)$ for $(\phi(g))(x)$. If $y \in E(X)$ and $g \in G$, then $g(o(y))=o(g(y))$, $g(t(y))=t(g(y))$, and $g(\bar{y})=\bar{g}(y)$. The case $g(y)=\bar{y}$ for some $g \in G$ and some $y \in E(X)$ may occur. That is, $G$ acts with inversions on $X$.

We have the following notations related to the action of the group $G$ on the graph $X$.

(1) If $x \in X$ (vertex or edge), we define $G(x)=\{g(x): g \in G\}$, and this set is called the orbit containing $x$.

(2) If $x, y \in X$, we define $G(x, y)=\{g \in G: g(x)=y\}$ and $G(x, x)=G_{x}$, the stabilizer of $x$. Thus, $G(x, y) \neq \varnothing$ if and only if $x$ and $y$ are in the same $G$ orbit. It is clear that if $v \in V(X), y \in E(X)$, and $u \in\{o(y), t(y)\}$, then $G(v, y)=\varnothing, G_{\bar{y}}=G_{y}$, and $G_{y} \leq G_{u}$.

3. The structure of groups acting on trees with inversions. In this section, we summarize the structure of groups acting on trees with inversions obtained by [4].

DEFINITION 3.1. Let $G$ be a group acting on a tree $X$, and $T$ and $Y$ two subtrees of $X$ such that $T \subseteq Y$. Then, $T$ is called a tree of representatives for the action of $G$ on $X$ if $T$ contains exactly one vertex from each $G$ vertex orbit, and $Y$ is called a fundamental domain for the action $G$ on $X$, if each edge of $Y$ has at least one end in $T$, and $Y$ contains exactly one edge $y$ (say) from each $G$ edge orbit such that $G(\bar{y}, y)=\varnothing$, and exactly one pair $x$ and $\bar{x}$ from each $G$-edge orbit such that $G(\bar{x}, x) \neq \varnothing$. It is clear that the properties of $T$ and $Y$ imply that if $u$ and $v$ are two vertices of $T$ such that $G(u, v) \neq \varnothing$, and if $x$ and $y$ are two edges of $Y$ such that $G(x, y) \neq \varnothing$, then $u=v$ and $x=y$ or $x=\bar{y}$.

Let $T$ and $Y$ be as above. Define the following subsets $Y_{0}, Y_{1}$, and $Y_{2}$ of edges of $Y$ as follows:

(1) $Y_{0}=E(T)$, the set of edges of $T$,

(2) $Y_{1}=\{y \in E(Y): o(y) \in V(T), t(y) \notin V(T), G(\bar{y}, y)=\varnothing\}$,

(3) $Y_{2}=\{x \in E(Y): o(x) \in V(T), t(x) \notin V(T), G(\bar{x}, x) \neq \varnothing\}$.

It is clear that $G$ acts with inversions on $X$ if and only if $Y_{2} \neq \varnothing$.

For the rest of this section, $G$ will be a group acting on a tree $X$ with inversions, $T$ will be a tree of representatives for the action of the group $G$ on $X$, 
and $Y$ will be a fundamental domain for the action of $G$ on $X$ such that $T \subseteq Y$. We have the following definitions.

DEFINITION 3.2. For each vertex $v$ of $X$, define $v^{*}$ to be the unique vertex of $T$ such that $G\left(v, v^{*}\right) \neq \varnothing$. That is, $v$ and $v^{*}$ are in the same $G$ vertex orbit. It is clear that if $v$ is a vertex of $T$, then $v^{*}=v$ and, in general, for any two vertices $u$ and $v$ of $X$ such that $G(u, v) \neq \varnothing$, we have $u^{*}=v^{*}$, and $G_{u}$ and $G_{v}$ are conjugate by an element of $G$. That is, for every element $b$ of $G_{u}$, there exist $g$ of $G$ and $a$ of $G_{v}$ such that $b=\operatorname{gag}^{-1}$.

DEFINITION 3.3. For each edge $y$ of $Y_{0} \cup Y_{1} \cup Y_{2}$, define $[y]$ to be an element of $G\left(t(y),(t(y))^{*}\right)$. That is, $[y]$ satisfies the condition that $[y]\left((t(y))^{*}\right)=t(y)$, and to be chosen as follows:

$$
[y]=1 \quad \text { if } y \in Y_{0}, \quad[y](y)=\bar{y} \quad \text { if } y \in Y_{2} .
$$

Define $[\bar{y}]$ to be the element

$$
[\bar{y}]= \begin{cases}{[y]} & \text { if } y \in Y_{0} \cup Y_{2}, \\ {[y]^{-1}} & \text { if } y \in Y_{1} .\end{cases}
$$

From above we see that $[y](y) \neq \bar{y}$ if $y \in Y_{0} \cup Y_{1}$ and $[y](y)=\bar{y}$ if $y \in Y_{2}$.

A group is termed a quasifree group if it is a free product of copies of $C_{\infty}$ and $C_{2}$, where $C_{\infty}$ denotes infinite cyclic group and $C_{2}$ a cyclic group of order 2 .

The following are examples of quasifree groups:

(1) every free group is a quasifree group. That is, a free product of copies of $C_{\infty}$ and a zero number of copies of $C_{2}$;

(2) the group of the presentation $\left\langle x, y, z \mid z^{2}=1\right\rangle \cong C_{\infty} * C_{\infty} * C_{2}$ is a quasifree group;

(3) the infinite dihedral group $\left\langle x, y \mid x^{2}=1, y^{2}=1\right\rangle \cong C_{2} * C_{2}$ is a quasifree group.

LEMMA 3.4. Let $G, X, Y$, and $T$ be as above such that the stabilizer of each vertex of $X$ is trivial. Then, $G$ is a quasifree group.

Proof. By [4, Theorem 3.6], $G$ has the presentation

$$
\begin{gathered}
\left\langle G_{v}, y, x\right| \operatorname{rel} G_{v}, G_{m}=G_{\bar{m}}, y \cdot[y]^{-1} G_{y}[y] \cdot y^{-1}=G_{y}, \\
\left.x \cdot G_{x} \cdot x^{-1}=G_{x}, x^{2}=[x]^{2}\right\rangle
\end{gathered}
$$

via the map $G_{v} \rightarrow G_{v}, y \rightarrow[y]$, and $x \rightarrow[x]$ where $v \in V(T), m \in Y_{0}, y \in Y_{1}$, and $x \in Y_{2}$.

Since the stabilizer of each vertex of $X$ is trivial, $G_{v}, G_{m}, G_{y}$, and $G_{x}$ are trivial for all $v \in V(T), m \in Y_{0}, y \in Y_{1}$, and $x \in Y_{2}$. This implies that $[x]^{2}=1$ for all $x \in Y_{2}$. Then, $G$ has the presentation $\left\langle y, x \mid x^{2}=1\right\rangle$, where $y \in Y_{1}$ and $x \in Y_{2}$. Then, $G$ is a free product of $C_{\infty}$ generated by $y$, and $C_{2}$ generated by $x$. 
This implies that $G$ is a quasifree group. This completes the proof.

COROLlary 3.5. Let $G, X, Y$, and $T$ be as above, and $H$ a subgroup of $G$ such that $H \cap G_{v}$ is trivial for all $v \in V(X)$. Then $H$ is a quasifree group.

DEFINITION 3.6. For each edge $y$ of $Y$, define the following.

(1) Define $-y$ to be the edge $-y=[y]^{-1}(y)$ if $o(y) \in V(T)$, otherwise $-y=y$.

(2) Define $+y$ to be the edge $+y=[y](-y)$.

It is clear that $t(-y)=(t(y))^{*}, o(+y)=(o(y))^{*}, G_{-y} \leq G_{(t(y))^{*}}$, and $G_{+y} \leq$ $G_{(o(y)) *}$. Moreover, if $y \in Y_{0} \cup Y_{2}$, then $G_{-y}=G_{+y}=G_{y}$.

(3) Define $\phi_{y}$ to be the map $\phi_{y}: G_{-y} \rightarrow G_{+y}$ given by $\phi_{y}(g)=[y] g[y]^{-1}$.

It is clear that $\phi_{y}$ is an isomorphism.

(4) Define $\delta_{y}$ to be the element $\delta_{y}=[y][\bar{y}]$.

It is clear that $\delta_{y}=1$ if $y \in Y_{0} \cup Y_{1}$, and $\delta_{y}=[y]^{2}$ if $y \in Y_{2}$. Consequently, $\delta_{y} \in G_{y}, \delta_{\bar{y}}=\delta_{y}$, and $\phi_{y}\left(\delta_{y}\right)=\delta_{y}$.

DEFINITION 3.7. By a word $w$ of $G$, we mean an expression of the form $w=g_{0} \cdot y_{1} \cdot g_{1} \cdot y_{2} \cdot g_{2} \cdots y_{n} \cdot g_{n}, n \geq 0, y_{i} \in E(Y)$, for $i=1,2, \ldots, n$ such that

(1) $g_{0} \in G_{\left(o\left(y_{1}\right)\right) *}$

(2) $g_{i} \in G_{\left(t\left(y_{i}\right)\right) *}$ for $i=1,2, \ldots, n$,

(3) $\left(t\left(y_{i}\right)\right)^{*}=\left(o\left(y_{i+1}\right)\right) *$ for $i=1,2, \ldots, n-1$.

If $w=1$, then $w$ is called a trivial word of $G$.

Let $w$ be the word defined above. We have the following concepts:

(a) $w$ is called reduced if $w$ contains no expression of the form $y_{i} \cdot g_{i} y_{i}^{-1}$ if $g_{i} \in G_{-y_{i}}$, or $y_{i} \cdot g_{i} \cdot y_{i}$ if $g_{i} \in G_{y_{i}}$ and $G\left(y_{i}, \bar{y}_{i}\right) \neq \varnothing$;

(b) we define $o(w)=\left(o\left(y_{1}\right)\right)^{*}$ and $t(w)=\left(t\left(y_{n}\right)\right)^{*}$. If $o(w)=t(w)=v$, then $w$ is called a closed word of $G$ of type $v$;

(c) the value of $w$ denoted by $[w]$ is defined as the element $[w]=$ $g_{0}\left[y_{1}\right] g_{1}\left[y_{2}\right] g_{2} \cdots\left[y_{n}\right] g_{n}$ of $G$

(d) $n$ is called the length of $w$ and is denoted by $|w|=n$;

(e) the inverse of $w$ denoted by $w^{-1}$ is defined as the word $w^{-1}=g_{n}^{-1} \cdot \bar{y}_{n}$. $\delta_{y_{n}}^{-1} g_{n-1}^{-1} \cdots g_{2}^{-1} \cdot \bar{y}_{2} \cdot \delta_{y_{2}}^{-1} g_{1}^{-1} \cdot \bar{y}_{1} \cdot \delta_{y_{1}}^{-1} g_{0}^{-1}$ of $G$.

It is clear that $\left[w^{-1}\right]=[w]^{-1}$ but $\left(w^{-1}\right)^{-1} \neq w$ if $w$ contains an edge $y$ (say) such that $G(y, \bar{y}) \neq \varphi$. Otherwise, $\left(w^{-1}\right)^{-1}=w$.

PROPOSITION 3.8. Every element of $G$ is the value of a closed and reduced word of $G$. Moreover, if $w$ is a nontrivial closed and reduced word of $G$, then $[w]$ is not the identity element of $G$. Moreover, if $w_{1}$ and $w_{2}$ are two closed and reduced words of $G$ such that $w_{1}$ and $w_{2}$ are of the same type and of the same value, then $\left|w_{1}\right|=\left|w_{2}\right|$.

Proof. See [5, Corollary 3.6].

4. The main result. The main result of this section is the following theorem. 
THEOREM 4.1. Let $G$ be a group acting on a tree $X$ with inversions such that each edge stabilizer is malnormal in $G$. Let $g$ be a nontrivial element of $G$ and $C(g)$ the centralizer of $g$ in $G$. Then,

(i) if $g$ is in a vertex stabilizer, then $C(g)$ is in that vertex stabilizer;

(ii) if $g$ is not in any vertex stabilizer, then $C(g)$ is an infinite cyclic subgroup of $G$ if $g$ does not transfer an edge of $X$ to its inverse. Otherwise, $C(g)$ is a finite cyclic subgroup of $G$ of order 2.

Proof. (i) Let $v$ be a vertex of $X$ such that $g \in G_{v}$. We need to show that $C(g)$ is contained in $G_{v}$. Let $f$ be an element of $G$ such that $f g=g f$. We need to show that $f$ is in $G_{v}$. We consider two cases.

CASE 1 ( $g$ is in $G_{x}$, where $x$ is an edge of $X$ such that $v$ is an end of $x$ ). Since the edge stabilizer for each edge of $X$ is malnormal in $G$, then $G_{X}$ is malnormal in $G_{v}$. This implies that $f$ is in $G_{x}$. Consequently, $f$ is in $G_{v}$ or, equivalently, $C(g)$ is contained in $G_{v}$.

CASE 2 ( $g$ is not in any edge stabilizer of $G$ ). Then, there exists a unique vertex $v^{*}$ of $T$ such that $G\left(v, v^{*}\right) \neq \varnothing$, that is, $v$ and $v^{*}$ are in the same $G$ vertex orbit. Then, there exist $a \in G$ and $b \in G_{v^{*}}$ such that $g=a b a^{-1}$. This implies that $v=a\left(v^{*}\right)$ and $a^{-1} f a b=b a^{-1} f a$. Let $h=a^{-1} f a$. Since $g \notin G_{x}$ for all $x \in E(X), t(x)=v$, therefore $h \notin G_{-y}$, for all $y \in E(Y)$ such that $(t(y))^{*}=$ $v$. By Proposition 3.8, there exists a reduced word $w=g_{0} \cdot y_{1} \cdot g_{1} \cdots y_{n} \cdot g_{n}$ of $G$ such that $w$ is of type $v^{*}$ and of value $h$. That is, $(o(y))^{*}=(t(y))^{*}=$ $v^{*}$ and $[w]=h$. Since $h \notin G_{-y_{n}}$, then $w \cdot h$ and $h \cdot w$ are reduced words of $G$ of value 1, the identity element of $G$. Therefore, by Proposition 3.8, the word $w \cdot h \cdot w^{-1} \cdot h^{-1}=g_{0} \cdot y_{1} \cdot g_{1} \cdots y_{n} \cdot g_{n} h g_{n}^{-1} \cdot \bar{y}_{n} \cdot \delta_{y_{n}}^{-1} g_{n-1}^{-1} \cdots g_{2}^{-1} \cdot \bar{y}_{2}$. $\delta_{y_{2}}^{-1} g_{1}^{-1} \cdot \bar{y}_{1} \cdot \delta_{y_{1}}^{-1} g_{0}^{-1} h^{-1}$ is not reduced. The only way that the indicated word can fail to be reduced is that $g_{n} h g_{n}^{-1} \in G_{-y_{n}}$. Replacing a subword of the form $y_{i} \cdot g_{i} \cdot \bar{y}_{i}$ if $g_{i} \in G_{-y_{i}}$ by $\phi_{y_{i}}\left(g_{i} \delta_{y_{i}}\right)$, or replacing a subword of the form $y_{i} \cdot g_{i} \cdot y_{i}$ if $g_{i} \in G_{y_{i}}$ and $G\left(y_{i}, \bar{y}_{i}\right) \neq \varnothing$, by $\phi_{y_{i}}\left(g_{i} \delta_{y_{i}}\right)$, we see that each $L_{i}=g_{n-i} \phi_{y_{n-i+1}}\left(L_{i-1}\right) g_{n-i}^{-1}$ is in $G_{-y_{n-i}}$ for $i=1, \ldots, n$ with the convention that $L_{0}=g_{n} h g_{n}^{-1}$ and $L_{n}=g_{0} \phi_{y_{1}}\left(L_{n-1}\right) g_{0}^{-1} h^{-1}=1$. Then $h=g_{0} \phi_{y_{1}}\left(L_{n-1}\right) g_{0}^{-1}$.

Since $\left(o\left(y_{1}\right)\right)^{*}=v^{*}, \phi_{y_{1}}\left(L_{n-1}\right) \in G_{+y_{1}} \leq G_{v^{*}}$, and $g_{0} \in G_{v^{*}}$, then $h \in G_{v^{*}}$. This implies that $a^{-1} f a \in G_{v}$. Therefore, $f \in a G_{v} a^{-1}=G_{v}$. Consequently $C(g)$ is contained in $G_{v}$.

(ii) Now, suppose that $g$ is not in any vertex stabilizer of $G$. Then, $C(g)$ has trivial intersection with each vertex stabilizer of $G$. If $a \neq 1$ is in $C(g)$, and $a$ is in a vertex stabilizer $G_{v}$ of $G$, for the vertex $v$ of $X$, then $g$ is in $C(a)$ and, by above, $g$ is in $G_{v}$. This contradicts the assumption that $g$ is not in any vertex stabilizer of $G$. Hence, by Corollary $3.5, C(g)$ is a free product of a number of infinite cyclic groups and a number of finite cyclic groups of order 2. Since $C(g)$ has a nontrivial center, and the center (see [3, Corollary 4.5, page 211]) of free product of groups of more than one factor is trivial, then $C(g)$ is an infinite cyclic groups, or $C(g)$ is a finite cyclic groups of order 2. If $g$ transfers an edge of $X$ to its inverse, then, by [7, Corollary 4.3], $C(g)$ is a finite cyclic 
group of order 2. Otherwise, $C(g)$ is an infinite cyclic group. This completes the proof.

DEFINITION 4.2. Let $n$ be a positive integer and $g$ a nontrivial element of the group $H$. We say that $g$ has at most $n$th root if whenever $g=a^{n}=b^{n}$, for $a, b$ in $H$, then $a=b$.

In the next corollaries, the group $G$ satisfies the hypothesis of Theorem 4.1.

COROLLARY 4.3. Any element of $G$ that is not in any vertex stabilizer of $G$ has at most $n$th root.

Proof. Let $g, a$, and $b$ be elements of $G$ such that $g$ is not in any vertex stabilizer of $G$, and $g=a^{n}=b^{n}$. We need to show that $a=b$. By Theorem 4.1, $C(g)$ is an infinite cyclic group or is a finite cyclic group of order 2 .

Since $g a=a g$ and $g b=b g$, then $a$ and $b$ are in $C(g)$. Then, it is clear that $g=a^{n}=b^{n}$ implies that $a=b$. This completes the proof.

COROLLARY 4.4. Let $g$ be an element of $G$. Then, $g$ is not in any vertex stabilizer of $G$ if and only if $g^{n}$ is not in any vertex stabilizer of $G$, where $n$ is a positive integer.

Proof. Since $g^{n}$ commutes with $g$, then $g^{n}$ is in $C(g)$ which, by Theorem 4.1 , is not in any vertex stabilizer of $G$ and the result follows. This completes the proof.

COROLLARY 4.5. Let $f$ and $g$ be two elements of $G$, and $m$ and $n$ two positive integers such that $f$ and $g$ are not in any vertex stabilizer of $G$ and $f^{m} g^{n}=$ $g^{n} f^{m}$. Then $f g=g f$.

Proof. From Corollary 4.4, we get

$$
\begin{aligned}
f^{m} g^{n}=g^{n} f^{m} & \Longrightarrow f^{m} g^{n} f^{-m}=g^{n} \\
& \Longrightarrow\left(f^{m} g f^{-m}\right)^{n}=g^{n} \\
& \Longrightarrow f^{m} g f^{-m}=g \\
& \Longrightarrow f^{m}=g f^{m} g^{-1} \\
& \Longrightarrow f^{m}=\left(g f g^{-1}\right)^{m} \\
& \Longrightarrow f=g f g^{-1} \\
& \Longrightarrow f g=g f .
\end{aligned}
$$

This completes the proof.

COROLLARY 4.6. Let $f$ and $g$ be two elements of $G$ such that $f$ and $g$ are not in any vertex stabilizer of $G$ and $f \in C(g)$. Then $C(f)=C(g)$. 
Proof. By Theorem 4.1, $C(f)$ and $C(g)$ are cyclic subgroups of $G$. Then, there exist two elements $a$ and $b$ of $G$ such that $a$ and $b$ are not in any vertex stabilizer of $G, C(f)=\langle a\rangle$, and $C(g)=\langle b\rangle$. It is clear that if $a \in C(g)$, then $C(f)=C(g)$. There exist two positive integers $m$ and $n$ such that $f=a^{m}$ and $g=b^{n}$. Since $f g=g f, a^{m} b^{n}=b^{n} a^{m}$. Then, Corollary 4.5 implies that $a b=b a$. This implies that $a b^{n}=b^{n} a$. Then $a \in C\left(b^{n}\right)=C(g)$. This completes the proof.

5. Applications. This section is an application of Theorem 4.1 and its corollaries. Free groups, free product of groups, free product of groups with amalgamation subgroup, tree product of groups, and HNN groups are examples of groups acting on trees without inversions. A new class of groups called quasi-HNN groups, defined in [2], are examples of groups acting on trees with inversions. In fact, free product of groups, free product of groups with amalgamation subgroup are special cases of tree product of groups and free groups and HNN groups are special cases of quasi-HNN groups.

Proposition 5.1. Let $G=\prod_{i \in I}^{*}\left(A_{i}, U_{j k}=U_{k j}\right)$ be a nontrivial tree product of the groups $A_{i}, i \in I$, such that $U_{i j}$ are malnormal subgroups of $G$. Let $g$ be a nontrivial element of $G$ and $C(g)$ be the centralizer of $g$ in $G$. Then,

(i) $C(g)$ is in a conjugate of $A_{i}$ for some $i, i \in I$, if $g$ is in a conjugate of $A_{i}$;

(ii) if $g$ is not in a conjugate of $A_{i}$, for all $i \in I$, then $C(g)$ is an infinite cyclic group and $g$ has at most $n$th root.

Proof. By [6], there exists a tree $X$ on which $G$ acts without inversions such that any tree of representatives for the action of $G$ on $X$ equals the corresponding fundamental domain for the action of $G$ on $X$, and for every vertex $u$ of $X$ and every edge $x$ of $X, G_{u}$ is isomorphic to $A_{i}, i \in I$, and $G_{x}$ is isomorphic to $U_{i k}$ for some $i, k$ in $I$. Moreover, $G$ contains no invertor elements. Therefore, by Theorem 4.1 and Corollary 4.3, the proof of Proposition 5.1 follows. This completes the proof.

COROLLARY 5.2. Let $G=\underset{C}{A} \underset{B}{B}$ be a free product of the groups $A$ and $B$ with amalgamation subgroup $C$ such that $C$ is a malnormal subgroup of $G$. Let $g$ be a nontrivial element of $G$ and $C(g)$ the centralizer of $g$ in $G$. Then,

(i) $C(g)$ is in a conjugate of $A$ or $B$ if $g$ is in a conjugate of $A$ or $B$;

(ii) if $g$ is not in a conjugate of $A$ or $B$, then $C(g)$ is an infinite cyclic group and $g$ has at most $n$th root.

COROLLARY 5.3. Let $G=A * B$ be a free product of the groups $A$ and $B$. Let $g$ be a nontrivial element of $G$ and $C(g)$ the centralizer of $g$ in $G$. Then,

(i) $C(g)$ is in a conjugate of $A$ or $B$ if $g$ is in a conjugate of $A$ or is in a conjugate of $B$;

(ii) if $g$ is not in a conjugate of $A$ or $B$, then $C(g)$ is an infinite cyclic group and $g$ has at most $n$th root. 
Proposition 5.4. Let $G^{*}$ be the quasi-HNN group

$$
G^{*}=\left\langle G, t_{i}, t_{j} \mid \operatorname{rel} G, t_{i} A_{i} t_{i}^{-1}=B_{i}, t_{j} C_{j} t_{j}^{-1}=C_{j}, t_{j}^{2}=c_{j}, i \in I, j \in J\right\rangle
$$

such that $A_{i}, B_{i}$, and $C_{j}, i \in I$ and $j \in J$, are malnormal subgroups of $G^{*}$. Let $g$ a nontrivial element of $G^{*}$ and $C(g)$ the centralizer of $g$ in $G^{*}$. Then,

(i) $C(g)$ is in a conjugate of $G$ if $g$ is in a conjugate of $G$;

(ii) if $g$ is not in a conjugate of $G$, then $C(g)$ is an infinite cyclic group or a finite cyclic group of order 2 and $g$ has at most $n$th root.

Proof. By [8, Lemma 5.1], there exists a tree $X$ on which $G^{*}$ acts with inversions such that $G^{*}$ is transitive on $V(X)$, and for every vertex $v$ of $X$ and every edge $x$ of $X, G_{v}^{*}$ is isomorphic to $G$ and $G_{x}^{*}$ is isomorphic to $A_{i}$, $i \in I$, or isomorphic to $C_{j}, j \in J$. Moreover, $G^{*}$ contains the invertor elements conjugate to an element $t_{j}, j \in J$. Therefore, by Theorem 4.1, the proof of Proposition 5.4 follows. This completes the proof.

COROLLARY 5.5. Let $G^{*}$ be the HNN group

$$
G^{*}=\left\langle G, t_{i} \mid \operatorname{rel} G, t_{i} A_{i} t_{i}^{-1}=B_{i}, i \in I\right\rangle
$$

such that $A_{i}$ and $B_{i}, i \in I$, are malnormal subgroups of $G^{*}$. Let $g$ a nontrivial element of $G^{*}$ and $C(g)$ the centralizer of $g$ in $G^{*}$. Then,

(i) $C(g)$ is in a conjugate of $G$ if $g$ is in a conjugate of $G$;

(ii) if $g$ is not in a conjugate of $G$, then $C(g)$ is an infinite cyclic group and $g$ has at most $n$th root.

COROLLARY 5.6. If $g$ is a nontrivial element of a free group $F$, then the centralizer $C(g)$ of $g$ in $F$ is an infinite cyclic group and $g$ has at most $n$th root.

ACKNOWLEDGMENT. The author would like to thank the referee for his sincere evaluation and constructive comments which improved the paper considerably.

\section{REFERENCES}

[1] A. Karrass and D. Solitar, The free product of two groups with a malnormal amalgamated subgroup, Canad. J. Math. 23 (1971), 933-959.

[2] M. I. Khanfar and R. M. S. Mahmood, On quasi HNN groups, J. Univ. Kuwait Sci. 29 (2002), no. 2, 13-24.

[3] W. Magnus, A. Karrass, and D. Solitar, Combinatorial Group Theory, Presentations of Groups in Terms of Generators and Relations, Dover Publications, New York, 1976.

[4] R. M. S. Mahmood, Presentation of groups acting on trees with inversions, Proc. Roy. Soc. Edinburgh Sect. A 113 (1989), no. 3-4, 235-241.

[5] _ The normal form theorem of groups acting on trees with inversions, J. Univ. Kuwait Sci. 18 (1991), 7-16.

[6] _ The subgroup theorem for groups acting on trees, Kuwait J. Sci. Eng. 25 (1998), no. 1, 17-33. 
[7] R. M. S. Mahmood and M. I. Khanfar, On invertor elements and finitely generated subgroups of groups acting on trees with inversions, Int. J. Math. Math. Sci. 23 (2000), no. 9, 585-595.

[8] _ Subgroups of quasi-HNN groups, Int. J. Math. Math. Sci. 31 (2002), no. 12, 731-743.

R. M. S. Mahmood: College of Education and Basic Science, Ajman University of Science and Technology, Abu Dhabi, United Arab Emirates

E-mail address: rasheedmsm@yahoo.com 


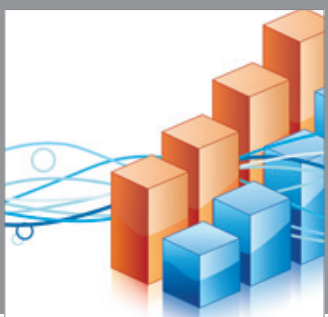

Advances in

Operations Research

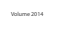

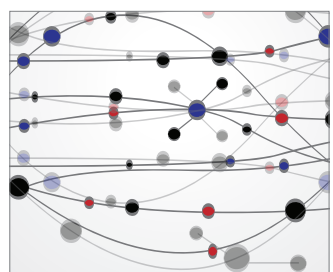

\section{The Scientific} World Journal
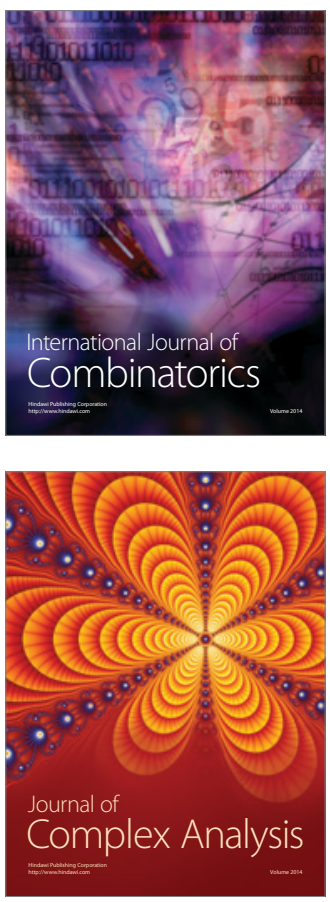

International Journal of

Mathematics and

Mathematical

Sciences
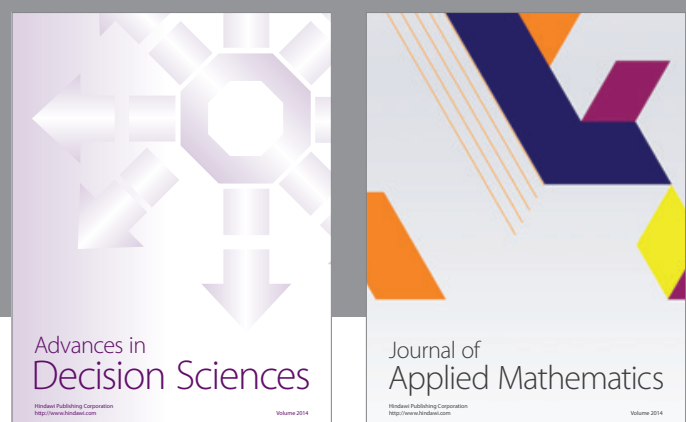

Journal of

Applied Mathematics
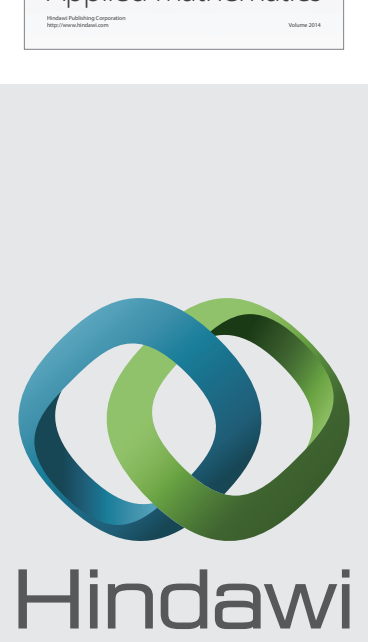

Submit your manuscripts at http://www.hindawi.com
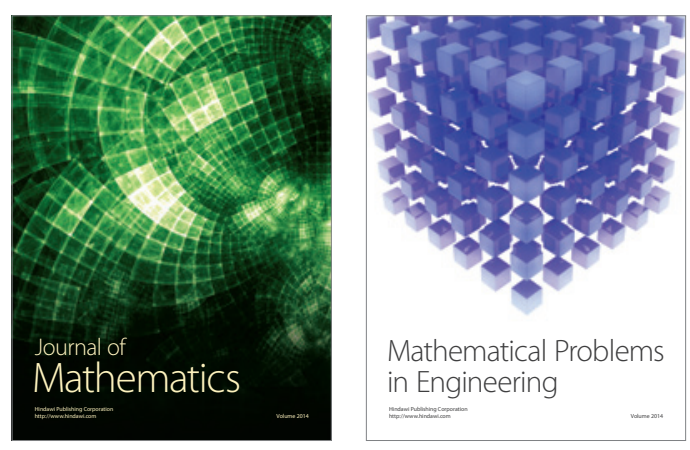

Mathematical Problems in Engineering
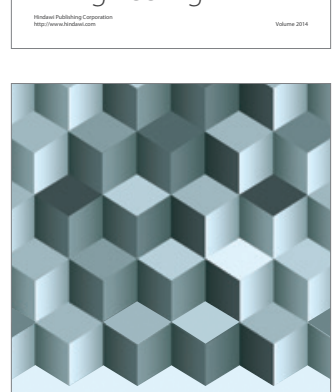

Journal of

Function Spaces
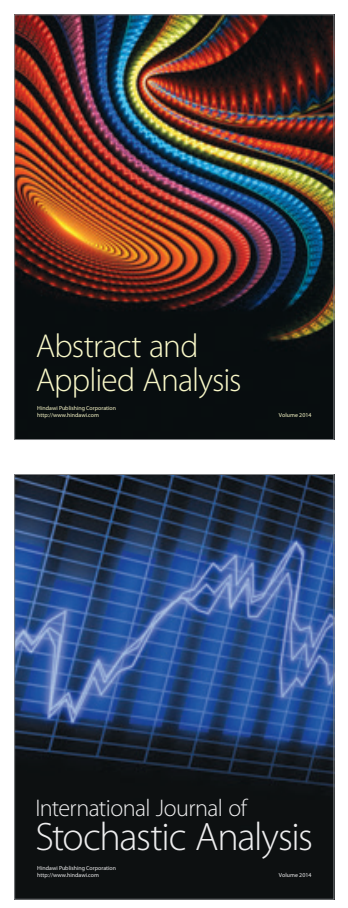

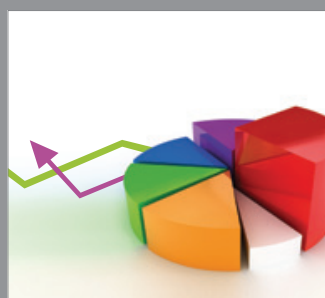

ournal of

Probability and Statistics

Promensencen
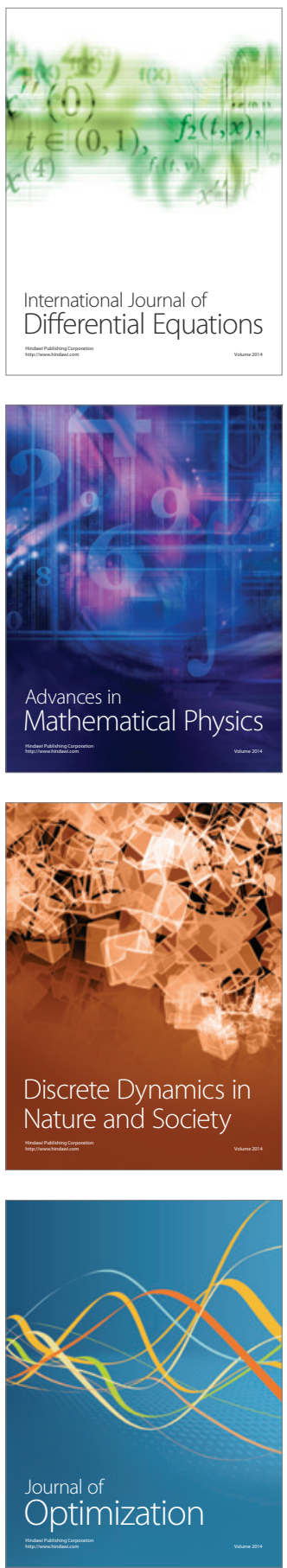